\title{
Institute for Mathematics and Democracy
}

\section{Stanley Chang, Andy Schultz, and Ismar Volić}

Mathematics has a well-deserved reputation as a vital instrument for understanding and improving our everyday experience. Math imbues the physics that controls the matter around us, the economics that explains our commercial markets, the algorithms that dictate our flow through the digital world. Despite its ubiquitous presence, however, there is one aspect of our lives in which mathematics is largely forgotten: politics.

Consider, for example, the most conspicuous of political events in the United States: presidential elections. The procedure for executing the election is multifaceted, with the Electoral College likely the best-known intermediary between vote-casting and the declaration of the winner.

But the Electoral College is not the linchpin of the electoral process; it is a single link in the chain that connects voter preferences to election results. Many other components of the presidential election process are just as important. What is the method by which the voters express their preferences? How is the winner in a given state determined? Why is each state granted its particular number of electoral votes? How does each state allocate those votes? How are voting districts determined?

Behind these questions lies the mathematics of voting, apportionment, and gerrymandering, subjects that are built upon combinatorics, probability, logic, statistics, geometry, dynamics, and many more fields. Additionally, mathematics governs much more than these basic

Stanley Chang is a cofounder of IMD and a professor of mathematics at Wellesley College. His email address is schang@we11 es 1 ey. edu.

Andy Schultz is an IMD affiliate and an associate professor of mathematics at Wellesley College. His email address is aschu1t2@we11es 1ey.edu.

Ismar Volic is a cofounder and director of IMD and a professor of mathematics at Wellesley College. His email address is ivo1ic@we11 es 1ey. edu.

Communicated by Notices Associate Editor Angela Gibney.

For permission to reprint this article, please contact: reprint-permission aams.org.

DOI: https://dx.doi.org/10.1090/noti2393 democratic processes; in the political arena itself, almost every public issue has a mathematical component (for example, healthcare, economy, and tax laws) and to a large extent can be understood and scrutinized quantitatively.

\section{Institute for Mathematics and Democracy}

The Institute for Mathematics and Democracy (IMD) ${ }^{1}$ was created to help cast light on the important role that mathematics plays in our political systems. Founded in 2019, it is housed at Wellesley College, a liberal arts institution with a strong interest in interdisciplinary work.

The mission of IMD is to promote a deeper understanding of the mathematics that underlies many socioeconomic forces and political processes so that citizens can make informed political decisions and effect meaningful change. It supports research, teaching, and outreach in mathematics and democracy, and brings together educators and activists who recognize the importance of political quantitative literacy. It acts as a facilitator, a repository, and nexus to bring research and pedagogy of disparate individuals who synthesize mathematics and democracy in their research and teaching.

The timeliness of these efforts is apparent as IMD is finding a wide and growing audience among students, researchers, educators, activists, and the public at large. Over thirty mathematicians now serve as scholars and affiliates for IMD, many of whom have pursued or supervised research, run summer programs, or created curricula at the intersection of politics and mathematics. The institute has also supported over twenty student researchers, almost half of them from underrepresented groups.

The activities of IMD are rooted and motivated by mathematics, but they lie at the unique interdisciplinary interface of mathematics, statistics, political science, economics, and history. Not only do they fill an evident educational and research gap, but they serve a social purpose: political

\footnotetext{
${ }^{1}$ IMD's webpage is https: //mathematics-democracy-institute.org/.
} 
quantitative literacy is one of the key tools for combating the crusade against science and reason that has been raging in recent years.

At the risk of artificially drawing lines between activities that are inherently interwoven, the next sections will elaborate on some of IMD's efforts in education, research, and outreach.

\section{Mathematics and Politics in the Classroom}

Quantitative literacy is one of the necessary ingredients for development and economic success. The educational system in the United States recognizes this need and in the last twenty or so years has generally adopted a significant quantitative literacy component, often embedded in STEM curricular initiatives.

However, political quantitative literacy has been largely neglected. The pedagogy of mathematics in the context of politics is largely missing from elementary, high school, and college curricula. Some problems with, say, plurality voting or the Electoral College might be studied through the prism of combinatorics in a civics or government class, or some misuses of statistics in politics might be given as examples in an introductory statistics course. This cross-disciplinary content, however, seems to be implemented exclusively by the initiative of individual teachers and professors.

The mathematics of politics is uniquely interdisciplinary and can be pitched at various levels, from middle school to advanced undergraduate or even graduate programs.

An example: Methods for tallying votes. Consider, for example, the task of gathering voter preferences and determining an election winner. When voters are expressing a preference between two candidates, the problem has a straightforward solution: a vote takes place, the ballots are tallied, and the winner is the candidate who receives the majority of votes. According to May's Theorem, this is indeed the "best" method for declaring the winner of a two-candidate election.

When there are more than two candidates, however, there are many ways in which preferences can be expressed and tallied. The most straightforward generalization of the process above is plurality voting where voters again express their top preference, with the winner being the candidate who receives the most votes.

However, there are also systems in which voters are allowed to rank (linearly) their preferences between candidates and then the winner is declared in some fashion. Some examples of these ranked choice voting methods are instant runoff, Borda count, and the Condorcet method (but there are many others).

For the instant runoff, a candidate who has a majority of the first-place votes is declared the winner. Otherwise, the candidate with the least number of first-place votes is eliminated, and, for each ranked ballot, their vote is transferred to the next candidate on the list. The procedure then runs iteratively until someone gets the majority of the votes. (For simplicity, we will ignore the possibility of a tie.)

For the Borda count, placements on each ballot are given a point value: if there are $n$ candidates, each first-place vote is worth $n-1$ points, each second-place vote is assigned $n-2$ points, and so on. Points are tallied and the candidate with the most points is the winner.

For the Condorcet method, ballots are used to determine winners of all possible head-to-head contests. If there is a candidate who beats all other candidates in these pairwise matchups, they are declared the winner.

A fascinating phenomenon is that these methods can produce different winners. For example, consider the ranked voting profile below, indicating that 36 voters listed the candidates as A, D, E, C, B in order of preference, 24 voters made the preference list $\mathrm{B}, \mathrm{E}, \mathrm{D}, \mathrm{C}, \mathrm{A}$, and so on: ${ }^{2}$

\begin{tabular}{cccccc}
36 & 24 & 20 & 18 & 8 & 4 \\
\hline A & B & C & D & E & E \\
D & E & B & C & B & C \\
E & D & E & E & D & D \\
C & C & D & B & C & B \\
B & A & A & A & A & A
\end{tabular}

One can now decide the winner using any of the procedures described above. It is clear that this election has no majority winner and that $\mathrm{A}$ is the plurality winner, but, as it turns out, $\mathrm{C}$ wins the instant runoff, $\mathrm{D}$ is the Borda count winner, and $\mathrm{E}$ is the Condorcet winner. (One can also use the rankings to find the winner of the usual runoff election where all but the top two scoring candidates are eliminated, and that happens to be B.)

These calculations require only rudimentary combinatorics and can be performed by a high school student. For a more advanced audience, the discussion now naturally leads to the attempt to determine the "best" method and to the introduction of the formalisms of social choice theory. The crowning conclusion of this line of investigation is the celebrated Arrow Impossibility Theorem, which states that the only ranked-choice voting method that satisfies a particular set of natural desirable fairness criteria is dictatorship-the system by which a single voter decides the outcome. The proof of Arrow's Theorem can easily be presented to undergraduates with a solid background in logic and combinatorics.

Other math and politics topics. A number of other topics lend themselves to such a layered instruction that can be adapted to various backgrounds. The basics of ranked and cardinal voting, the Electoral College, the efficiency gap and compactness scores in gerrymandering, power indices, and apportionment of legislative seats are all accessible to high school students. They can be contextualized at a higher level by introducing social choice theory and game theory,

\footnotetext{
${ }^{2}$ This example was taken from The Mathematics of Elections and Voting by W. H. Wallis.
} 
including the intricate impossibility theorems of Arrow, Gibbard-Satterthwaite, and Balinski-Young.

The Prisoner's Dilemma and Nash Equilibrium are just the first steps of the game-theoretic point of view, and many advanced themes can be framed and understood within this field (competition, conflict, strategy, fair division, corruption, bureaucracy). Linear algebra, abstract algebra, and probability can be used at various junctures.

There are many opportunities to enrich the material by employing statistics and data analysis that use current real-life election, census, or other datasets. Occasions to support the mathematics with examples from history, economics, and politics-many of them current and relevant-are also plentiful. These resources make the content especially attractive to students who are apprehensive about taking a "standard" math course.

Sample courseware. Although not ubiquitous in undergraduate mathematics departments, there have been some college-level courses on mathematics and politics that have successfully run at various institutions. Such a course at Wellesley College, ${ }^{3}$ for instance, has quickly become remarkably popular, attracting one of the most diverse audiences the mathematics department has ever seen. There are also efforts by a number of middle school and high school teachers to bring this content into their classrooms.

IMD has created a webpage that serves as a repository of mathematics and politics teaching materials for anyone who wishes to use them. ${ }^{4}$ The page links to class websites and other resources used by a number of college faculty, as well as lesson plans used by several middle school teachers around the country. The links lead to lecture notes, worksheets, problem sets, videos, datasets, and other materials. Already used by some middle school, high school, and college faculty, these resources help to mitigate a growing demand for guidance among those who wish to incorporate mathematics and politics into their teaching.

Given the remarkable appetite for these resources, IMD is constantly working to synthesize and improve them, creating a roadmap with ready-made teaching modules that can be inserted selectively into existing curricula or used as the basis for the creation of entire courses aimed at audiences at various levels.

\section{Research in Mathematics and Politics}

Research in mathematics and politics has thus far been largely performed by mathematics-adjacent economists and political scientists. There are of course notable exceptions in which mathematicians make significant contributions to voting theory or the larger fields of social choice and game

${ }_{3}^{3}$ See https://sites.google.com/wellesley.edu/mathematicsand politicsspring21.

${ }^{4}$ See https://mathematics-democracy-institute.org/education -resources/ theory. But in general, the intersection of mathematics and politics is not on display as an apparent and available research direction in the mathematical community.

However, research in this area that is initiated by mathematicians has been increasing in visibility in recent years. More mathematicians are supervising undergraduate research and thesis projects in topics like voting theory and gerrymandering. Programs like the math and politics REU at Grand Valley State University, and organizations such as the Metric Geometry and Gerrymandering Group point to the possibilities of successfully employing abstract mathematics to obtain new and deep results, often in combination with cutting-edge techniques in data analysis. Journals that are friendly to mathematically inclined work in social sciences, such as Mathematical Social Sciences and Social Choice and Welfare, are becoming more familiar to mathematicians.

Indeed, the interdisciplinarity and the complexity of the subjects that reside in the intersection of mathematics and politics give rise to rich opportunities for research at the professional and undergraduate levels. Not only is this observation evident by the variety and the quality of the work of the IMD affiliates, but also by the research performed by students under the direction of IMD over the last two years.

Some of the IMD student research topics include representation theory on tabloids and positional voting, a category-theoretic interpretation of the Balinski-Young Theorem, combinatorial topology models for political structures, Banzhaf and Shapley-Shubik power indices via simplicial complexes, and a graph-theoretic approach to liquid democracy. Several of these projects will or have already resulted in publications. The participation of underrepresented groups in this research was encouragingly high, again attesting to the interdisciplinary appeal of the kind of mathematics that is outside the traditional curricular path and is additionally timely and relevant.

In response to an ever-growing demand for research opportunities in mathematics and politics, IMD has taken on various roles in facilitating research interactions and supporting research projects. IMD's faculty run a yearlong Undergraduate Fellows Program that has a significant research component, and organize an eight-week REU-like intensive summer research program. IMD also acts as an intermediary between faculty who are already engaged in this work and interested students; in parallel with our webpage for educational resources, we are developing an online compendium of research topics and materials, along with contacts and descriptions of research backgrounds of faculty who are willing to work with students.

\section{Outreach, Events, and Opportunities}

Outreach. Because of its immediate real-world applicability and the increased visibility of topics like gerrymandering and ranked-choice voting, there is an appetite for the 
discussion of the role of mathematics and politics beyond the traditional academic venues, namely classrooms and scholarly publications.

IMD affiliates have given talks to various groups, e.g., high schools and political activist organizations, to help increase awareness of these important topics. They have also disseminated this message more broadly through a variety of op-eds, and even supported ballot measures that promote mathematically sound voting practices.

Special events. IMD has organized a number of events, lectures, and conferences. Here are two representative ones:

On Election Night in 2020, IMD Fellows and faculty held a virtual event for the general public to explain the mathematical intricacies and flaws of the Electoral College, various voting methods, and the Congressional apportionment process.

In May 2021, IMD organized a one-day virtual conference, Math and Politics: Numeracy at the Ballot Box. Lectures were presented by middle school, college, and university faculty and affiliates. The speakers recounted their experiences teaching mathematics and politics, running a math and politics REU program, performing research in mathematics and politics with students, and being a mathematician in the world of politics. Several undergraduate students presented their research, and one high school student codelivered a lecture.

Both events were exceptionally well-attended. The recordings of the presentations are accessible on the IMD website. ${ }^{5}$

Opportunities. There are many opportunities for mathematicians to foster political quantitative literacy. Fortunately, expertise in the subject is not a prerequisite for participation. IMD hopes to act as a facilitator in this area for interested educators and scholars.

Mathematicians who wish to become conversant with the field might want to review some educational resources listed on IMD's website. Those resources also cater to those who are interested in a deeper investigation and wish to teach an introductory course on the subject at their school, reach out to adjacent disciplines (e.g., political science, economics, data science) to identify colleagues who might have room for a special lecture by a mathematician, or who might even want to collaborate on a course.

For those interested in a less intense debut in the subject, the perspective can be flipped: instead of teaching a course which examines the mathematics in political processes, one could instead bring political processes into the standard mathematics courses. For example, the Condorcet method discussed above allows one to convert the slate of ballots

${ }^{5}$ For more details on the 2020 Election Night event, see https:// mathematics-democracy-institute.org/election-night-event -videos-and-presentations/, and on the May 2021 conference, see https://mathematics-democracy-institute.org/2021 -conference/

into a "real-world" directed graph. Examples of mathematics and politics in other standard mathematics subjects can be found by reviewing the courseware at the IMD website.

For those readers who might be interested in participating in the emerging intersection between mathematics and politics, IMD wants to help. The institute welcomes all interested parties to collaborate on projects and participate in upcoming events.

\section{The Urgency of Getting Involved}

The practice of politics is contentious and messy. Having a solid understanding of the mathematics that lies beneath political processes will not necessarily resolve this; if anything, regarding these processes through a mathematical lens confirms that complexity is fundamental to their nature.

On the other hand, by ignoring the mathematics behind politics, we leave our democratic institutions vulnerable. Democracies have always relied on a meaningfully educated electorate to operate most effectively, and political quantitative literacy is a skill that can no longer be allowed to languish.

We hope our mathematics community will recognize the necessity of playing an active role in advancing political quantitative literacy and the gravity of the repercussions if we fail to do so. The Institute of Mathematics and Democracy is committed to making more mathematicians aware of this increasingly important application of our subject and helping them incorporate it into their professional lives.

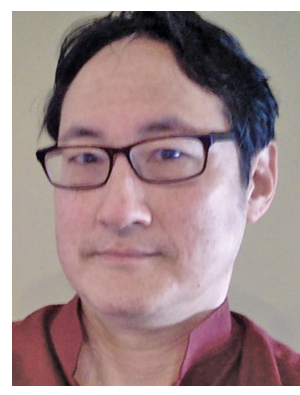

Stanley Chang

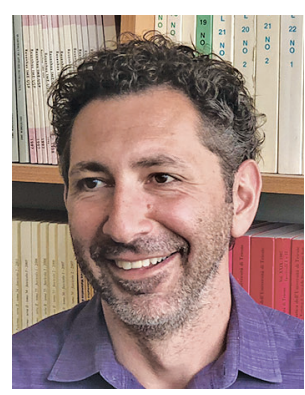

IsmarVolić

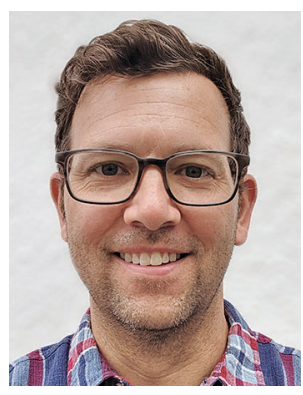

Andy Schultz

\section{Credits}

Photo of Stanley Chang is courtesy of Stanley Chang.

Photo of Andy Schultz is courtesy of Andy Schultz.

Photo of Ismar Volić is courtesy of Franjo Šarčević. 